\title{
ORIGINAL ARTICLE \\ Incomplete offspring sex bias in Australian populations of the butterfly Eurema hecabe
}

\author{
DJ Kemp ${ }^{1,2}$, FE Thomson ${ }^{2}$, W Edwards ${ }^{2}$ and I Iturbe-Ormaetxe ${ }^{3,4}$
}

Theory predicts unified sex ratios for most organisms, yet biases may be engendered by selfish genetic elements such as endosymbionts that kill or feminize individuals with male genotypes. Although rare, feminization is established for Wolbachiainfected Eurema butterflies. This paradigm is presently confined to islands in the southern Japanese archipelago, where feminized phenotypes produce viable all-daughter broods. Here, we characterize sex bias for E. hecabe in continental Australia. Starting with 186 wild-caught females, we reared >6000 F1-F3 progeny in pedigree designs that incorporated selective antibiotic treatments. F1 generations expressed a consistent bias across 2 years and populations that was driven by an $\sim 5 \%$ incidence of broods comprising $\geqslant 80 \%$ daughters. Females from biased lineages continued to overproduce daughters over two generations of outcrossing to wild males. Treatment with antibiotics of differential strength influenced sex ratio only in biased lineages by inducing an equivalent incomplete degree of son overproduction. Brood sex ratios were nevertheless highly variable within lineages and across generations. Intriguingly, the cytogenetic signature of female karyotype was uniformly absent, even among phenotypic females in unbiased lineages. Molecular evidence supported the existence of a single Wolbachia strain at high prevalence, yet this was not clearly linked to brood sex bias. In sum, we establish an inherited, experimentally reversible tendency for incomplete offspring bias. Key features of our findings clearly depart from the Japanese feminization paradigm and highlight the potential for more subtle degrees of sex distortion in arthropods.

Heredity (2017) 118, 284-292; doi:10.1038/hdy.2016.85; published online 12 October 2016

\section{INTRODUCTION}

Sex in most diploid organisms is diecious and anisogamous, and produces sons and daughters in a balanced 1:1 ratio. Theoretically, Fisher's principle (Fisher, 1930) explains how selection should stabilize sex ratios at unity to maximize the inclusive fitness gain from parental investment. This principle is based on a form of negative frequencydependent selection, wherein the ultimate genetic payoff of investing in sons versus daughters depends on the frequency of each gender in the population. More formally, a unified offspring sex ratio represents the evolutionarily stable strategy (Maynard Smith, 1982) at equilibrium. Adaptive departures from unity sometimes occur, either at population levels as in parthenogenic and haplodiploid species, or at brood levels according to parental investment theory (for example, Trivers and Willard, 1973). The expectation for most species is nevertheless for a unified ratio, and departures at equilibrium present opportunities to explore the assumptions of sex allocation theory (Hamilton, 1967).

In recent decades, sex ratio has been intensively studied across a range of arthropod groups with regard to the effects of obligate microbial endosymbionts such as Rickettsia (Himler et al., 2011), Spiroplasma (Jiggins et al., 2000), Cardinium (Zchori-Fein and Perlman, 2004) and Wolbachia (Werren, 2008). Such microbes exist within host cells and achieve vertical transmission only by 'hitch-hiking' in the cytoplasm of female gametes. This means that the evolutionary capacity to invade and occupy host populations hinges upon realized rates of maternal inheritance (Cordaux et al., 2011). Investigation across a broad range of endosymbionts has revealed the ability to manipulate host reproduction in ways commensurate with this goal. Four primary manipulations are known (Werren, 2008; Cordaux et al., 2011). First, thelytokous parthenogenesis is restricted to haplodiploid hosts and causes female rather than male phenotypes to develop from unfertilized gametes. Second, cytoplasmic incompatibility (hereafter, 'CI') renders matings between uninfected males and infected females inviable; hence reducing the effective population contribution of uninfected males. Third, male killing promotes the selective death of sons in embryonic or juvenile stages (Hurst et al., 1999), and may elevate the marginal fitness of infected daughters owing to factors such as reduced inter-sibling competition (Hurst and Majerus, 1993). Fourth, feminization causes genetically male hosts to develop reproductively viable female phenotypes. This manipulation is rarely documented, yet offers high evolutionary payoffs (in conventionally diploid arthropod hosts) by doubling the potential number of conduits for vertical transmission. Although not always maladaptive for hosts, these manipulations often create misalignments of evolutionary interests that, in turn, set the scene for host-endosymbiont coevolution (Riegler and O'Neill, 2007; Cordaux et al., 2011). Documented host counteradaptations include nuclear

${ }^{1}$ Department of Biological Sciences, Faculty of Science and Engineering, Macquarie University, Sydney, New South Wales, Australia; ${ }^{2}$ Centre for Tropical Environmental Sustainability Science and College of Science and Engineering, James Cook University, Cairns, Queensland, Australia and ${ }^{3}$ School of Biological Sciences, The University of Queensland, Brisbane, Queensland, Australia

Correspondence: Dr DJ Kemp, Department of Biological Sciences, Faculty of Science and Engineering, Macquarie University, North Ryde, Sydney, New South Wales 2109, Australia.

E-mail: darrell.kemp@mq.edu.au

${ }^{4}$ Current address: Institute of Vector-borne Disease, Monash University, Clayton, Victoria, Australia.

Received 3 November 2015; revised 30 June 2016; accepted 25 July 2016; published online 12 October 2016 
suppressors that restore the viability and/or natural phenotypic development of male genotypes (Hornett et al., 2006; Charlat et al., 2007b).

Lepidoptera have proven an excellent group for exploring arthropodendosymbiont dynamics (Dyson and Hurst, 2004; Hornett et al., 2006; Sugimoto and Ishikawa, 2012). This group harbors bacterial symbionts such as Spiroplasma and Wolbachia collectively known to impose CI, male killing and feminization (Jiggins et al., 2000; Hiroki et al., 2004; Charlat et al., 2005). Studies of butterfly male-killing have yielded valuable insights into host-symbiont coadaptation, including the genetic and biogeographic basis of nuclear host suppression (Dyson and Hurst, 2004; Hornett et al., 2006, 2008; Charlat et al., $2007 b, c)$. Lepidopterans have also informed the study of feminization, primarily via the work in Wolbachia-infected Ostrinia moths (Crambidae) and Eurema butterflies (Pieridae). Whereas sex bias in Ostrinia was initially interpreted as feminization, subsequent work has revealed a more complex effect based ultimately upon male killing (Kageyama and Traut, 2004; a manipulation also induced by Spiroplasma in this genus; Tabata et al., 2011). Data from extensive historic and contemporary studies of Eurema (outlined below) have nevertheless remained consistent with feminization. This system poses a rare example of this phenomenon in the Arthropoda (Cordaux et al., 2011), which outside of isopods is known for a mite (Weeks et al., 2001), a leafhopper (Negri et al., 2006) and a wasp (Giorgini et al., 2009).

Evidence for Eurema feminization has arisen from studies of Japanese E. hecabe/mandarina. The effect was first established for E. mandarina (then considered a subspecies of E. hecabe; Hiroki, 2002; Hiroki et al., 2004; Narita et al., 2007a, c), but has since been extended to the species distinguished as E. hecabe (Narita et al., 2011). Each species is subject to infection by the same two genetically distinct strains of Wolbachia. The first strain $(w C I)$ occurs at near fixation in Eurema populations throughout Japan (Narita et al., 2011). It has been established to impose strong cytoplasmic incompatibility across this range, but is also known for male killing in a single mainland population (Narita et al., 2007b). The second strain ( $w$ Fem) is restricted to islands in the southern Japanese archipelago, where it occurs as a low frequency coinfection alongside wCI (Hiroki, 2002; Hiroki et al., 2004; Narita et al., 2011). Doubly infected females from these populations are known to produce broods consisting entirely of daughters, yet produce $100 \%$ sons if treated with antibiotics (Hiroki, 2002; Hiroki et al., 2004). This implies that such individuals in fact possess male sexual karyotypes and are capable of engendering normal development of male phenotypes in the absence of feminization. Cytogenetic study has corroborated this view because doubly infected phenotypes lack the signature of female (W) chromatin readily visualized in genetic females (Hiroki, 2002; Narita et al., 2007a, 2011). More recently, Kern et al. (2015) used Z-linked genetic markers to reveal that doubly infected female E. mandarina possess just one paternally inherited $\mathrm{Z}$ chromosome, and raised the possibility that $w$ Fem may mimic $\mathrm{W}$ chromosomes in such individuals.

This study examines sex ratio distortion in E. hecabe on the Australian continent. Offspring bias is not presently known for this species beyond Japan, nor has it been noted in Australian Eurema despite decades of research across the genus (for example, Jones et al., 1987; Jones, 1992). Interestingly, more recent pedigree-based study of E. hecabe from tropical North Queensland revealed the overproduction of daughters in a small fraction of lineages (DJK, unpublished data). Although offspring biases may arise for diverse reasons (Hurst et al., 1996), the paradigm established in Japanese conspecifics lends precedent to a hypothesis of Wolbachia causality. The most straightforward extension of this paradigm offers two basic scenarios. The first is that Australian butterflies experience feminization simply in the manner of their oriental counterparts. This would imply a Western Pacific distribution of $w \mathrm{Fem} / w \mathrm{CI}$ that extends (or has recently extended) well beyond the known limits of subtropical Japanese islands (for example, Narita et al., 2011). The second is that either or both strains (or their variants) reside in Australia but engender male killing (as seen rarely in Japan; Narita et al., 2007b). Either case would present opportunities to examine the broader biogeographic and/or coevolutionary context of host-symbiont interaction. Of course, sex distortion could arise from any number of variants on these scenarios, or entirely different scenarios involving novel Wolbachia strains, other symbionts and microorganisms (Kageyama et al., 2012), or nuclear factors such as epistasis and meiotic drive (Jiggins et al., 1999; Weeks et al., 2002).

We first aimed to establish the incidence of offspring sex bias in Australian E. hecabe, as expressed among broods of two tropical populations obtained over successive years. We next sought to test whether sex biases exhibit the maternal inheritance and antibiotic sensitivity characteristic of endosymbiotic bacteria. Experimental antibiotic treatments were applied selectively to lineages each year to test whether broods revert either to unity (1:1), as indicative of male killing, or to the all-male phenotypes evidenced in feminized Japanese conspecifics (Narita et al., 2011). Last, we explored offspring sexual karyotypes using cytogenetic assays and investigated the presence of Wolbachia (specifically, the strains $w \mathrm{CI}$ and $w$ Fem) using diagnostic PCR and sequencing.

\section{MATERIALS AND METHODS}

\section{General husbandry protocols}

We used standard husbandry protocols throughout. All work was conducted in a single climate-controlled laboratory (temperature $=26.0 \pm 1.0^{\circ} \mathrm{C}$, humidity $=$ $60 \pm 5 \%$, photoperiod $=14 \mathrm{~L}: 10 \mathrm{D}$ (2008) and 16L:8D (2009)). Overhead fluorescent and metal-halide lights provided bright, full-spectrum illumination (Kemp, 2008) and hostplant (Sesbania cannabina) was grown in an outdoor greenhouse.

Lines were established each year using females captured at Cairns $\left(16.90^{\circ} \mathrm{S}\right.$, $\left.145.75^{\circ} \mathrm{E}\right)$ and Port Douglas (16.48 $\left.\mathrm{S}, 145.46^{\circ} \mathrm{E}\right)$ in North Queensland, Australia. Females were stored in $60 \mathrm{ml}$ specimen jars with access to $15 \%$ honey water, and at $\sim 9: 00 \mathrm{~h}$ the next day, placed individually in blindly labeled oviposition cups containing a hostplant cutting (Figure S1). This was repeated across days until females had laid $>35$ eggs or expired, when they were frozen at $-20^{\circ} \mathrm{C}$. Hatchlings developed communally until the fourth and fifth instars, when they were separated into groups of 8-10 and 4-5, respectively. Hostplant was provided throughout in oversupply. Adults were sexed using the presence of male-limited ventral androconial brands.

Females designated as dams for subsequent generations were marked with identifying numbers on both ventral hindwings (using colored Sharpie markers). They were released along with an equivalent number of males into $600 \mathrm{~mm}^{3}$ mating cages (aluminium-fiberglass mesh construction; Kemp, 2008). The cages were monitored at $15 \mathrm{~min}$ intervals during light hours to identify mating pairs, which were removed to individual gauze-topped containers (Figure S1).

\section{Breeding experiment}

This experiment was designed to inform the incidence and magnitude of sex bias, establish whether it is inherited and provide preliminary insight into antibiotic effects. We started by rearing $1449 \mathrm{~F} 1$ offspring from females caught at Cairns $(N=45 ; 14$ February 2008) and Port Douglas $(N=38 ; 13$ February 2008; Figure 1a). At eclosion, we identified the 10 most female-biased families, mated daughters from each family with random wild-caught males and reared their offspring to adulthood. Twenty F1 females produced a total of $160 \mathrm{~F} 2$ individuals (Figures $1 \mathrm{~b}$ and $\mathrm{c}$ ). Following eclosion, we randomly assigned daughters from the three most female-biased F2 families among two treatments (Figure 1c): 
(1) Control: housed for 3 days in a gauze-topped cup (Figure S1) with ad libitum access to a cotton-wool bud soaked with $15 \%$ honey water;

(2) Treatment: housed as controls but with $1 \mathrm{mg}$ tetracycline hydrochloride supplemented per $1 \mathrm{ml}$ of honey water (as shown to invert sex ratio in Japanese Eurema; Hiroki, 2002; Hiroki et al., 2004; Narita et al., 2007b).

Females were subsequently mated to wild-caught males and used to rear an F3 generation (comprising $N=229$ adults from 13 control dams and 217 adults from 15 treatment dams; Figure 1d).

\section{Breeding experiment}

This experiment expanded upon the 2008 design by nesting antibiotic treatment within lineages designated as either female-biased or unbiased (1:1) sex ratio. We also added a third dam feeding treatment based on rifampicin, which is a bacteriocidal compound of greater potency than tetracycline (Charlat et al., 2007a). Females caught from Cairns $(N=63)$ and Port Douglas $(N=40)$ on 27 February 2009 (Figure 2a) were used to rear 1661 F1 adults. Daughters from each of five female-biased and five unbiased lineages were randomly assigned to control $(\mathrm{C})$, tetracycline $(\mathrm{T})$ or rifampicin $(\mathrm{R})$ feeding treatments (Figure 2b). The latter treatment consisted of both tetracycline and rifampicin dissolved in honey water, each at a concentration of $1 \mathrm{mg} \mathrm{ml}^{-1}$. Females were subsequently mated with wild-caught males and contributed an F2 generation comprising 2354 total offspring (Figure 2c). Finally, we undertook a limited series of crosses between control F2 individuals (Figure 2d) to further investigate the inheritance of sex bias; these are outlined in the results.

\section{Molecular assays}

Diagnostic PCR assays were used to test the presence of Wolbachia DNA in selected experimental subjects (specified in the results) in both years. Single butterflies were homogenized for $90 \mathrm{~s}$ in a $2 \mathrm{ml}$ screw cap tube containing three sterile glass beads $(2 \mathrm{~mm})$ and $600 \mu \mathrm{l}$ of squash buffer $(10 \mathrm{~mm}$ Tris, $1 \mathrm{~mm}$ EDTA, 50 mм EDTA, pH 8.2), using a bead beater (Biospec, Bartlesville, OK, USA). The homogenate was centrifuged for $10 \mathrm{~min}$ at $20000 \mathrm{~g}$ and the DNA was extracted from the supernatant using phenol/chloroform/isoamyl alcohol followed by a chloroform/isoamyl alcohol, and precipitated using sodium acetate and ethanol. DNA was resuspended in $50 \mu \mathrm{l}$ TE buffer, and $1 \mu \mathrm{l}$ of a $1 / 20$ dilution was used for the PCR. We used the forward primer wsp $81 \mathrm{~F}$ and the reverse primer wsp691R to amplify a portion of the wsp gene, which codes

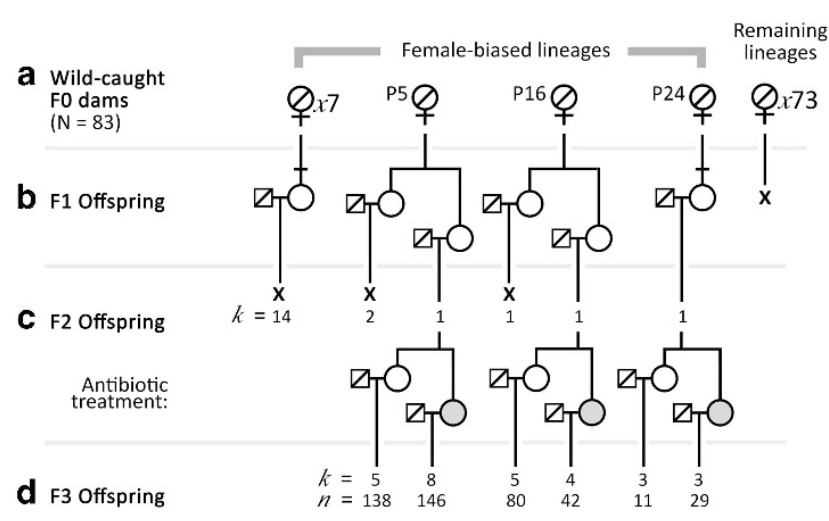

Figure 1 Schematic for the 2008 experiment. Dams (circles) and sires (squares) are depicted with a diagonal slash if wild-caught, and duplicate crosses are collapsed to aid presentation. (a) Wild-caught females contributed an $\mathrm{F} 1$ generation of 1449 individuals in $k=83$ families. (b) Daughters from the 10 most female-biased F1 families were then outcrossed with wild-caught males; (c) F2 offspring ( $n=160$ in $k=20$ broods) were reared to adulthood. Daughters from three female-biased families (tracing from F0 lineages 'P5', 'P16' and 'P24') were randomly assigned to control versus tetracycline (shaded) feeding treatments, then outcrossed with wild males; (d) F3 offspring (total $n=446$ in $k=28$ broods) were subsequently reared for the assessment of adult sexual phenotype. for an outer membrane protein in Wolbachia (Zhou et al., 1998). We also tested for the presence of $w \mathrm{CI}$ and $w \mathrm{Fem}$, the two strains known for Japanese $E$. hecabe (Narita et al., 2011), using the following primer pairs:

(1) wsp81F (5'- TGGTCCAATAAGTGAAGAAAC - 3') (Zhou et al., 1998) and $w$ HecFem1 (5'-ACTAACGTCGTTTTTGTTTAG-3') (Hiroki et al., 2004) that amplify a $232 \mathrm{bp}$ section of the $w s p$ gene characteristic of strain $w \mathrm{CI}$;

(2) $w$ HecFem2 (5'-TTACTCACAATTGGCTAAAGAT-3') (Hiroki et al., 2004) and wsp691R (5'-AAAAATTAAACGCTACTCCA-3') (Zhou et al., 1998) that amplify a $398 \mathrm{bp}$ section of the wsp gene characteristic of $w$ Fem.

To confirm successful DNA extraction in each sample, we also performed PCRs using the $28 \mathrm{~S}$ ribosomal DNA primer set (28sF3633+28sR4076; RugmanJones et al., 2010) or $18 \mathrm{~S}$ ribosomal DNA (18sF+18sR; Duplouy et al., 2013), both of which amplify insect DNA. All molecular work was undertaken at the University of Queensland's Wolbachia laboratory using a proof-reading Elongase mix (Life Technologies, Carlsbad, CA, USA), following the manufacturer's instructions. PCR cycling conditions were as follows: $95^{\circ} \mathrm{C}$ for $3 \mathrm{~min}$, followed by $95^{\circ} \mathrm{C}$ for $30 \mathrm{~s}, 53{ }^{\circ} \mathrm{C}$ for $30 \mathrm{~s}$ and $68^{\circ} \mathrm{C}$ for $1 \mathrm{~min}$ for $35 \mathrm{cycles}$, then $68^{\circ} \mathrm{C}$ for $10 \mathrm{~min}$. PCR products were run on agarose gels and stained with ethidium bromide. PCR products obtained used the primer combination $w s p 81 \mathrm{~F}+w \mathrm{HecFem} 1$ or $w s p 691 \mathrm{R}+w \mathrm{HecFem} 2$, were purified using a gel extraction kit (Qiagen, Hilden, Germany), cloned into pGEM-Teasy (Promega, Madison, WI, USA) and sequenced at AGRF using T7 primers.

\section{Cytogenetic assays}

Sexual karyotype was investigated using a standard cytogenetic technique for visualizing female $\mathrm{W}$ chromatin (Traut et al., 2007). We assayed male and female phenotypes from selected lineages and as fully specified in the results. Unfortunately, live Japanese conspecifics were not available for comparison, however, we assayed several additional butterfly species (Belenois java; Pieridae and Hypolimnas bolina; Nymphalidae) as positive controls for the technique.

\section{Statistical procedures}

We conducted discrete contrasts of sex ratio according to the absolute frequencies of males and females. Exact binomial tests were used to assess departures from unity (that is, the nominal null hypothesis for butterflies), and likelihood-based G-tests were used to test 'intrinsic' hypotheses for sex ratio

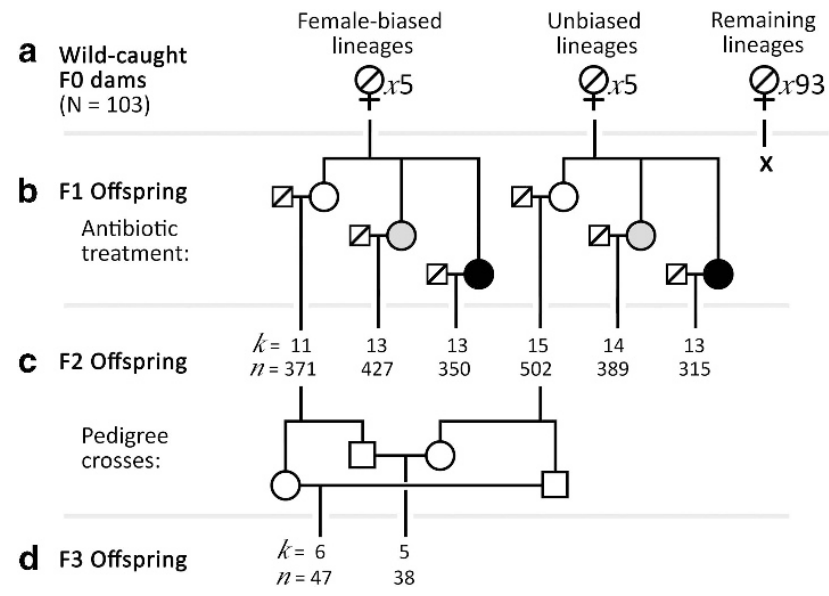

Figure 2 Schematic for the 2009 experiment, presented as per Figure 1. (a) We reared the broods of 103 wild females, and identified 5 femalebiased and 5 unbiased F1 families; (b) Daughters in each F1 family were randomly assigned to control, tetracycline or rifampicin feeding treatments (open, shaded and solid circles, respectively), then outcrossed with wildcaught males; (c) F2 offspring ( $n=2354$ in $k=79$ broods) were reared to adulthood. Finally, we performed reciprocal crosses between biased and unbiased control F2 individuals, and (d) reared F3 offspring ( $n=85$ in $k=11$ broods). 
variation among different groups. The aim to assess biases among multiple individual broods introduced the potential for type II error; hence, we conducted such tests using replicated goodness of fit procedures (Sokal and Rohlf, 1995; pp. 715-724). Overall goodness of fit (to a 1:1 ratio) was partitioned into components owing to deviance for all offspring pooled versus deviance contributed by individual broods. Significantly biased broods were then identified using the a posteriori simultaneous test procedure.

General linear models were used to analyze the experimental data sets in each year. We tested main and interactive effects, as well as a priori contrasts for specific treatment differences in 2009, as specified below. All models analyzed daughter frequency (dependent variable) as a function of total brood size (random covariate); hence, sex ratio was parameterized as the marginal frequency of daughters. This was desirable because broods varied greatly in offspring number and simple sex ratio indices (such as \% daughters) inflated the variance contribution of smaller families. Data of daughter and offspring frequencies were normalized prior to analysis using the square-root transformation, which is appropriate for data consisting of counts (Sokal and Rohlf, 1995).

All models included treatment as a fixed effect, and in 2009, included sex ratio pedigree (that is, whether broods derived from putatively 1:1 or femalebiased pedigree). We also tested in both years for differences among broods of different F0 lineage, specified as a fixed factor because lineages were chosen $a$ priori (Sokal and Rohlf, 1995). In 2009, we specified three planned contrasts for the effect of antibiotic treatment on F2 sex ratio. The first was designed to test prediction for no effect of experimental group in 1:1 lines (that is, $\mathrm{C} \sim \mathrm{T} \sim \mathrm{R}$ ).

Table 1 Summary of F1 offspring of female $E$. hecabe caught from Cairns and Port Douglas in each study year

\begin{tabular}{|c|c|c|c|c|c|c|}
\hline \multirow{2}{*}{$\begin{array}{l}\text { Year } \\
\text { Sampling population }\end{array}$} & \multicolumn{3}{|c|}{2008} & \multicolumn{3}{|c|}{2009} \\
\hline & Cairns & Port Douglas & Total & Cairns & Port Douglas & Total \\
\hline Wild-caught (FO) dams & 45 & 38 & 83 & 63 & 40 & 103 \\
\hline \multicolumn{7}{|l|}{ F1 offspring } \\
\hline Males & 318 & 324 & 642 & 472 & 311 & 783 \\
\hline Females & 408 & 399 & 807 & 521 & 357 & 878 \\
\hline Total & 726 & 723 & 1449 & 993 & 668 & 1661 \\
\hline F1 sex ratio (\%female) & 56.2 & 55.2 & 55.7 & 52.5 & 53.4 & 52.9 \\
\hline
\end{tabular}

The next two contrasts were designed to test specific effects in female-biased lines: (a) for divergence of controls from both treatment groups (that is, $\mathrm{C} \neq \mathrm{T}$ $+\mathrm{R}$; where we predicted female bias in $\mathrm{C}$ ) and (b) for divergence among treatment groups (that is, $\mathrm{T} \neq \mathrm{R}$ ).

All analyses were performed using Statistica version 7.1 (Statsoft, Tulsa, OK, USA). Means are reported with standard errors throughout, with sex ratio described as \%females (that is, $\mathrm{F} /(\mathrm{M}+\mathrm{F})$ ) unless otherwise specified.

\section{RESULTS}

\section{Incidence of offspring sex bias in Australia}

Wild-caught females produced F1 offspring comprising 642 sons versus 807 (55.7\%) daughters from 83 broods in 2008, and 783 sons versus 878 (52.9\%) daughters from 103 broods in 2009 (Table 1). Overall, sex ratio exhibited a female bias in each year (exact binomial $P<0.0001$ (2008) and $P<0.05$ (2009)), which did not vary among years $\left(G_{1}=2.50, P=0.114\right)$ or populations (that is, Cairns versus Port Douglas; $G_{1}=0.150, P=0.698$ (2008); $G_{1}=0.153, P=0.696$ (2009)).

Sex ratio varied significantly among $F 1$ broods in both 2008 $\left(G_{82}=145.0, \quad P<0.0001\right)$ and $2009 \quad\left(G_{102}=176.3, \quad P<0.0001\right)$. The simultaneous test procedure identified five 2008 broods as significantly biased. These comprised between 79.2 and $90.0 \%$ daughters $($ mean $=85.0 \pm 4.8 \%$; Figure 3$)$. Five biased broods were identified in 2009, of which four were female-biased (range $=78.8-100 \%$ daughters; mean $=87.1 \pm 9.5 \%$ ) and one comprised $92.3 \%$ sons (Figure 3 ). Only one of $186 \mathrm{~F} 1$ broods across the sample consisted entirely of daughters.

\section{Breeding experiment}

In 2008, we reared offspring from 10 female-biased F1 lineages (Table 2). The overall F2 sex ratio deviated from unity (exact $P<0.0001)$ and more greatly than evidenced for the broader F1 generation (that is, $65.6 \%$ versus $55.7 \%$ females; $G_{1}=5.90, P<0.05$ ). This supports a maternal component of inheritance because the nonrandom selection of F1 dams engendered a more strongly female-biased offspring generation. The degree of bias was however weaker than expressed across the 10 contributing F1 families (that is, the broods from which founding dams were selected; $G_{1}=5.17$, $P<0.05)$. Although F2 family sizes precluded broader analysis, there was no categorical evidence for the production of $100 \%$ daughters. Sex

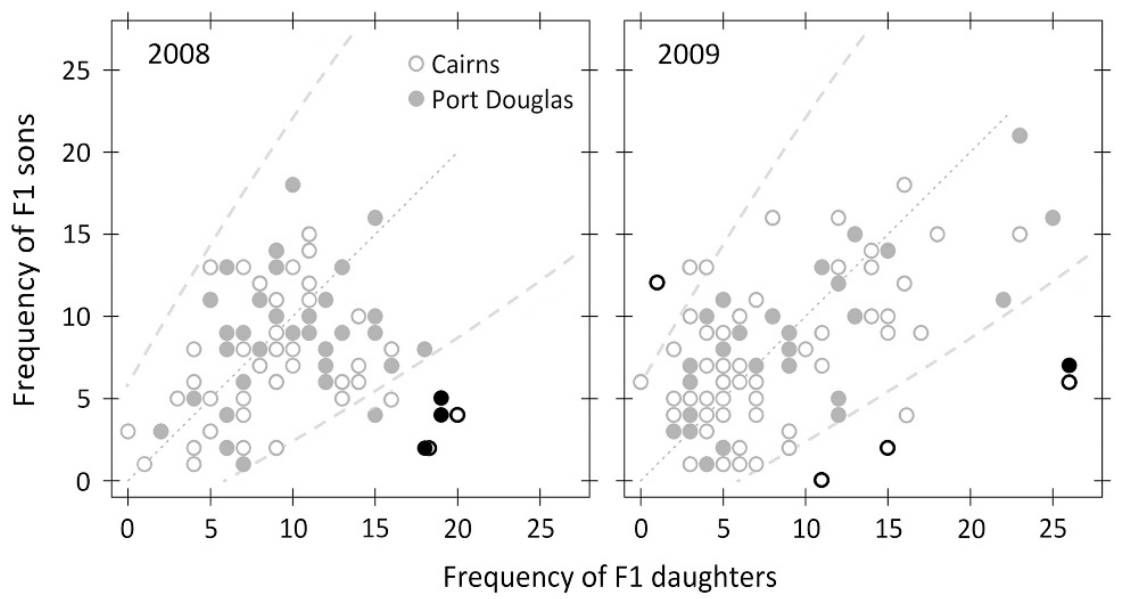

Figure 3 Frequencies of sons versus daughters among the broods of wild-caught females in 2008 and 2009 . Each point represents a single F1 family. Points are presented in halftone except those determined as deviating significantly from 1:1 sex ratio (the dotted central line) according to the replicated goodness of fit G-test. The outer dashed lines indicate the $95 \%$ confidence bounds for binomially expected outcomes under a hypothesis of $1: 1$ sex ratio, which is a less statistically conservative criterion for detecting bias. 
ratio was instead varied considerably, even within lineages (as shown notably by the two large broods in the 'P5' lineage that comprised 52\% versus 92\% daughters; Table 2).

We reared an F3 generation using dams from three female-biased families (tracing from three separate lineages) that were assigned across control (honey water) versus treatment (tetracycline) groups. General linear models analysis revealed that brood sex ratio was affected by feeding treatment $\left(F_{1,21}=26.2, P<0.001\right)$, but did not

Table 2 Sex ratios of F1 and F2 broods in the 10 lineages that were perpetuated for the $\mathbf{2 0 0 8}$ experiment (labelled according to FO dam ID)

\begin{tabular}{lllll}
\hline Lineage ID & $F 1$ offspring & \multicolumn{3}{c}{$F 2$ broods } \\
\cline { 2 - 4 }$M: F(\% F)$ & $N$ & $M: F(\% F)$ & Sum \\
\hline
\end{tabular}

(a) Port Douglas population

$\begin{array}{lcccc}\text { P5 } & 5: 19(79.2)^{\mathrm{a}} & 3 & 2: 24(92.3) & 14: 36(72.0) \\ & & 9: 10(52.6) & \\ & & 3: 2(60.0) & \\ & & & 3: 7(70.0) & 6: 14(70.0) \\ \text { P14 } & 4: 19(82.6)^{\mathrm{a}} & 3 & 2: 1(87.5) & \\ & & & 1: 16(94.1) & 2: 16(88.9) \\ & & & 1: 0(0.0) & \\ \text { P16 } & 1: 7(87.5) & 2 & 13: 12(48.0) & 16: 13(44.8) \\ & & & 3: 1(25.0) & \\ \text { P19 } & 7: 16(69.6) & 2 & 1: 4(80.0) & 3: 4(57.1) \\ & & & 2: 0(0.0) & \\ \text { P21 } & 4: 15(78.9)^{\mathrm{b}} & 2 & 2: 9(81.8) & 2: 9(81.8) \\ & & & 2: 3(60.0) & 3: 4(57.1) \\ \text { P24 } & 8: 12(60.0) & 1 & 1: 1(50.0) & \end{array}$

(b) Cairns population

$\begin{array}{lcccc}\text { C15 } & 7: 16(69.6) & 1 & 1: 0(0.0) & 1: 0(0.0) \\ \text { C25 } & 4: 20(83.3)^{\mathrm{a}} & 3 & 3: 3(50.0) & 4: 5(55.5) \\ & & 0: 2(100) & \\ & & & 1: 0(0.0) & \\ \text { C20 } & 6: 14(70.0) & 1 & 4: 4(50.0) & 4: 4(50.0) \\ \text { Sum } & 48: 156(76.5) & 20 & & 55: 105(65.6)\end{array}$

Abbreviations: ' $\mathrm{M}$ ' = male, ' $\mathrm{F}$ ' = female. Lineages highlighted in bold type contributed to subsequent generations. For $\mathrm{F} 1$ broods.

${ }^{\text {a }} P<0.05$ according to the simultaneous test procedure.

${ }^{\mathrm{b}} P<0.05$ according to the exact binomial test. differ among lineages $\left(F_{2,21}=0.235, P=0.793\right)$. There was no significant interaction between treatment and lineage $\left(F_{2,21}=0.238\right.$, $P=0.790)$; hence, the effect of treatment was consistent across all lineages. In sum, control F3 offspring expressed a female bias (79 sons: 150 daughters; exact $P<0.0001$ ), whereas treatment offspring were male-biased (147 sons: 70 daughters overall; exact $P<0.0001$; Figure 4a). The absolute departure from 1:1 was indistinguishable among groups $\left(G_{1}=0.251, P=0.616\right)$, which suggests that antibiotic treatment inverted an otherwise heritable female bias to an equivalent degree of son overproduction (Figure 4a).

As in previous generations, there was no evidence for $100 \%$ sex-biased offspring. Treated F2 dams exclusively produced more sons than daughters, but brood sex ratios spanned a practical range of 20-45\% females (Figure 5; see Table S1 for full details). Control offspring expressed a similar spread of female bias except for one brood in the 'P5' lineage comprising 30 sons versus 13 (30.2\%) daughters. This contrasted notably against two clearly female-biased broods generated by control sisters (for example, 5:32 and 6:24 sons: daughters; Figure 5). Overall, control offspring exhibited a weaker female bias than their founding F2 families (that is, 65.5\% versus $90.7 \%$ females; $\left.G_{1}=15.8, P<0.0001\right)$.

\section{Breeding experiment}

In 2009, we reared an F2 generation using dams of 1:1 versus femalebiased $\mathrm{F} 1$ pedigree that were allocated across control, tetracycline and rifampicin treatments. General linear models analysis indicated that F2 brood sex ratio did not vary according to pedigree $\left(F_{1,72}=1.53\right.$, $P=0.220)$, but there was an effect of treatment $\left(F_{2,72}=8.00\right.$, $P<0.001)$, and an effect of the interaction between pedigree and treatment $\left(F_{2,72}=3.30, P<0.05\right.$; Figure $\left.4 \mathrm{~b}\right)$. The significant interaction effect revealed that sex ratio responded differently to antibiotic treatment in the female-biased versus the 1:1 pedigree. We further informed the nature of this effect via three planned contrasts. The first contrast revealed no effect of antibiotic treatment among broods of unbiased pedigree $(t=0.915, P=0.363)$. Offspring sex ratio did not deviate from unity either within each experimental group or across all groups pooled (control: exact $P=0.177$; tetracycline: $P=0.384$; rifampicin: $P=0.630$; all groups: $P=0.976)$. The remaining two contrasts dealt only with the female-biased pedigree, indicating that: (a) brood sex ratios in both treatment groups deviated from controls $(t=4.86$, $P<0.0001)$ and (b) sex ratios were equivalent under tetracycline

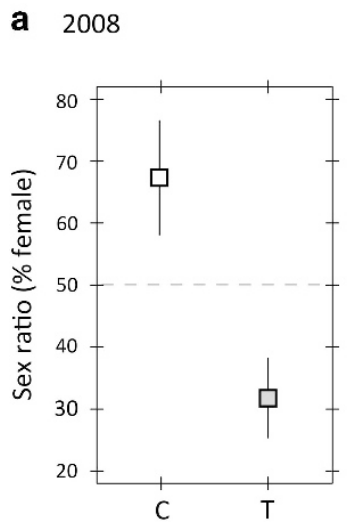

b 2009

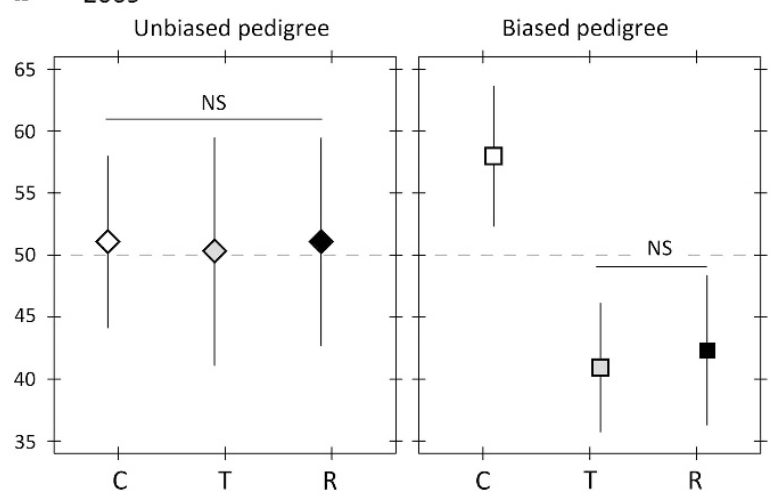

Figure 4 Summary of experimental antibiotic effects for (a) F3 offspring in 2008 and (b) F2 offspring in 2009. Dams of either female-biased (squares) or unbiased (diamonds) pedigree were assigned across control $(C)$, tetracycline $(T$; halftone) and rifampicin ( $R$; solid) treatment groups. Mean $\pm 95 \%$ confidence interval for sex ratio is indicated. Groups within panels differed significantly $(P<0.05)$ except as shown for 2009. NS, not significant. 
versus rifampicin treatment $(t=0.305, P=0.762)$. As in 2008, control broods retained a female bias (exact $P<0.0001$ ), but antibiotic treatment induced male bias (tetracycline: exact $P<0.001$; rifampicin: $P<0.005)$. Additional analyses conducted separately for each pedigree group indicated no significant variation among constituent lineages and no significant treatment $x$ lineage interaction (details provided in Table S2).
F2 broods in this experiment again provided no indication for $100 \%$ sex biases (Figure 6; Tables S3-S4). Control broods of sex-biased pedigree (Figure 6a) also echoed earlier results by indicating a weaker female bias than their founding $\mathrm{F} 1$ families $\left(G_{1}=26.07\right.$, $P<0.0001)$.

Last, we performed limited crosses between control F2 individuals to inform the paternal inheritance of sex bias. Full siblings from four

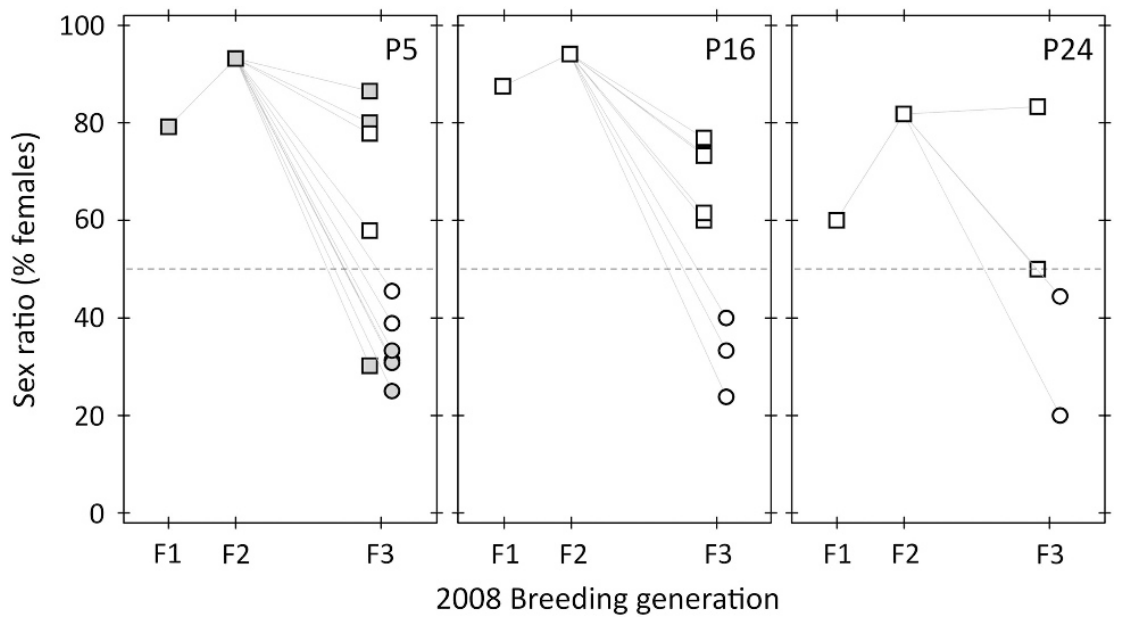

Figure 5 Brood offspring sex ratios across each of three lineages perpetuated in the 2008 experiment. Squares indicate broods derived from unmanipulated (honey water-fed) dams and circles indicate F3 broods derived from tetracycline-fed dams. Panels are labeled for matriline identity (and trace to Table 2). Shaded broods in matriline 'P5' contributed individuals for diagnostic Wolbachia PCR assays and sequencing.

a Female-biased lineages:

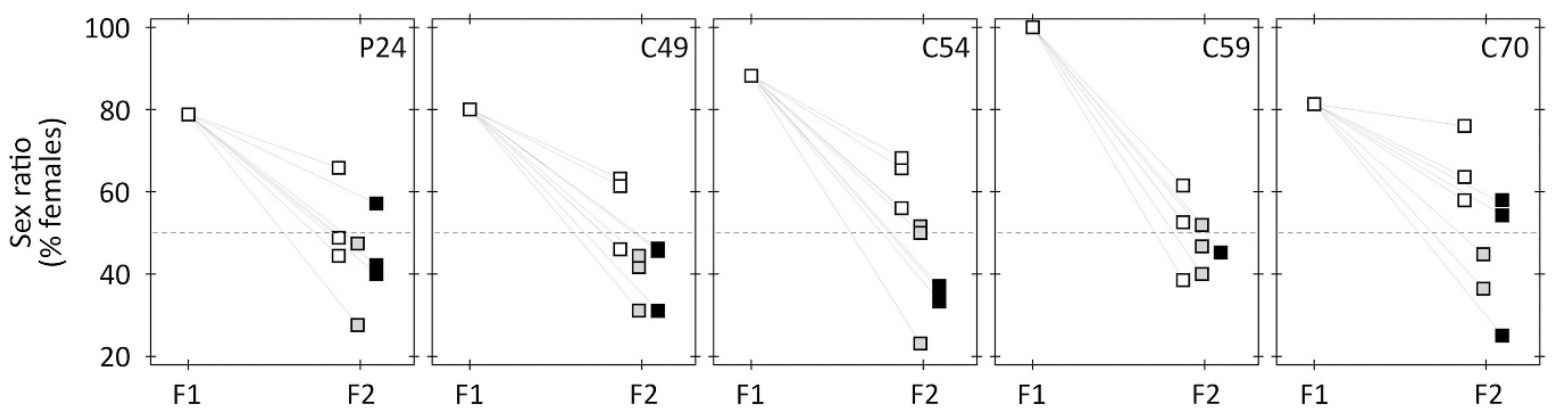

b Unbiased (1:1) lineages:

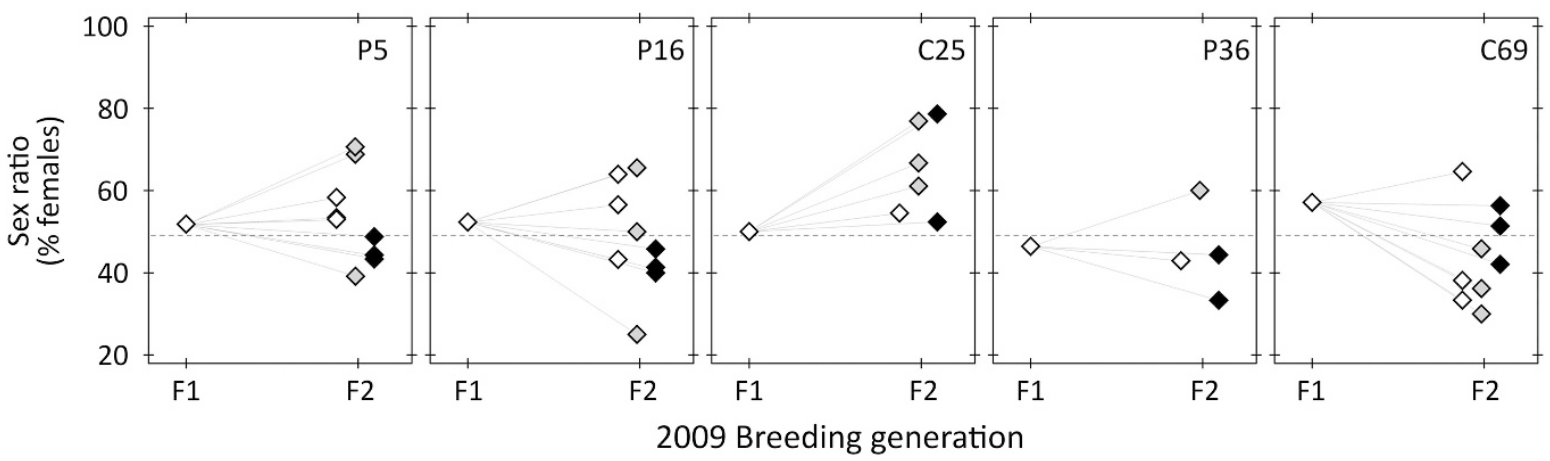

Figure 6 Brood sex ratios across the F1 and F2 generations for (a) female-biased and (b) unbiased lineages in the 2009 experiment (indicated as squares and diamonds, respectively). Symbols indicate broods from untreated (open), tetracycline-treated (shaded) and rifampicin-treated (solid) F1 dams. Panels are labeled according to matriline identity. Brood sex ratios are fully outlined in Tables S3-S4. 
Table 3 Overview of F2 crosses performed in 2009

\begin{tabular}{lcccc}
\hline$F 2$ cross details & & F3 Offspring \\
\hline Parental lineages (total \%F) & Total broods & M:F (\%F) & Deviation from unity (1:1) & Deviation from parental bias \\
\hline Biased sires (65.9) $\times 1: 1$ dams (55.7) & 5 & $21: 17(44.7)$ & $P=0.627$ & $G_{1}=6.16, P<0.05$ \\
$1: 1$ sires (56.7) $\times$ biased dams (65.5) & 6 & $19: 28(59.6)$ & $P=0.243$ & $G_{1}=0.585, P=0.444$ \\
\hline
\end{tabular}

Sex ratio, given as \%females, is summed for the F2 families from which sires and dams were chosen, and also for F3 broods obtained from each group of crosses (a full breakdown of individual broods is provided by Supplementary Table S5). Deviation from unity (1:1) was assessed using exact binomial tests.

biased families were used as sires and dams in crosses with offspring from several unbiased lineages (as indicated by Figure $2 \mathrm{c}$ and detailed in Table S5). Overall, F3 broods obtained from sex-biased sire crosses comprised $44.7 \%$ daughters, whereas those obtained from sex-biased dam crosses comprised 59.6\% daughters. Neither sex ratio deviated significantly from unity; however, sires but not dams failed to engender the strength of female bias expressed among founding F2 lineages (Table 3).

\section{Molecular assays}

In 2008, we screened for the presence of Wolbachia DNA among individuals in the ' $\mathrm{P} 5$ ' lineage according to the sampling scheme shown by Figure 5. We tested an F1 female, six of her F2 daughters (three control and three tetracycline-treated, post-oviposition), and five F3 male and female offspring per daughter. All 67 tested individuals were seen to amplify positively for Wolbachia DNA. This included the three control F2 daughters and their progeny, even despite highly divergent F3 brood sex ratios. More notably, this also encompassed tetracycline-treated F2 daughters and their progeny across three male-biased F3 broods (Figure 5). Wolbachia DNA was detected in all of 16 wild females sampled in 2009, which included the 5 dams that produced biased progeny, (Figure 3) as well as 11 that produced unbiased broods. Nine rifampicin-treated F2 females and four untreated F2 females of biased pedigree likewise tested positive. Strain-specific PCRs amplified positively for $w C I$, but never $w$ Fem in all tested specimens across both years. Cloned 602 bp sequences of the wsp gene obtained from all 67 individuals in 2008 proved $100 \%$ identical to $w C I$.

\section{Cytogenetic assays}

In 2009, we examined over 60 male and female phenotypes from groups of 1:1, female-biased and male-biased F2 broods (the latter two groups arising from control versus antibiotic-treated lineages of sex-biased pedigree). In no case did we detect the $\mathrm{W}$-chromatin characteristic of female karyotype. The assay however proved $100 \%$ accurate for sexual phenotype in B. java and H. bolina (Figure S2).

\section{DISCUSSION}

Eurema butterflies are an exemplar for genetic feminization, yet this paradigm is confined to a cluster of Japanese Islands (Narita et al., 2011). Here we reveal the incidence of sex distortion well beyond this biogeographic range. We show that a small fraction of Australian E. hecabe produce incompletely female-biased broods, a feature that is both maternally heritable and disrupted by antibiotic treatment. Although consistent with the action of Wolbachia, we only detected one (non-feminizing) Japanese strain. We also found no evidence of female (WZ) karyotypes in any individual, which is noteworthy, given the prominence of cytogenetic assays in supporting lepidopteran feminization. In sum, our findings implicate bacteria as an agent of sex bias, yet neither demonstrate nor discount the role of Wolbachia, and clearly depart from the Japanese paradigm.

\section{Incidence of offspring sex bias}

Studies of lepidopteran sex distortion have largely addressed malekilling effects, where female bias occurs at or close to $100 \%$ (for example, Hurst et al., 1999; Jiggins et al., 2000; Charlat et al., 2005). Complete brood biases have also been reported virtually without exception in feminized Eurema (Hiroki, 2002; Hiroki et al., 2004; Narita et al., 2011). Against this background, our findings are notable in revealing fractional degrees of daughter overproduction. This was evident among broods obtained from wild-caught females (Figure 3), as well as F2-F3 offspring across treatment groups (discussed below). The total observed incidence of all-female F1 broods (1/186) contrasts starkly with reports for feminized E. mandarina (3/14 and 2/10; Hiroki, 2002; Hiroki et al., 2004) and E. hecabe (3/15; Narita et al., 2011).

The continuous nature of offspring sex ratio posed important analytical consequences. Most broadly, it relegated judgements of bias to statistical inference, and thereby dependent upon sample size and subject to sampling variance. Power for concluding bias scaled with offspring number (and was effectively 0 for $n<10$ ). This implies the likelihood of imprecision in our assignment of biased F1 broods. Further, a reliance on statistical criteria inevitably conflates estimation of within-brood bias strength (that is, average effect size) with the population incidence of such broods. Whereas we conclude a conservative $5 \%$ incidence of $\sim 85 \%$ female F1 broods, more relaxed statistical criteria identify nearly twice the number of slightly less-biased broods (indicated by Figure 3). Ultimately, this disposed a greater emphasis on analyses at lineage and pedigree/group levels.

\section{The inheritance of bias}

We perpetuated each years' pedigree by outcrossing females with wildcaught males, which prioritized the test of maternal inheritance. At the group level, biased lineages indicated daughter overproduction across all generations, whereas unbiased lineages engendered 1:1 offspring. This established the inheritance of sex bias through maternal lines, a critical assumption of endosymbiont causality (Werren, 2008; Cordaux et al., 2011). Although our design precluded a detailed assessment of paternal effects (discussed further below), several crosses in 2009 supported a stronger vector of maternal inheritance.

Although variation at brood levels offered a limited basis for (a posteriori) statistics, several points were notable. First, there was inconsistency among lineages in whether and how strongly F1 sex bias carried through to the F2 generation (for example, lines P19, P32 and C25; Table 2). This contrasts with the perfect inheritance of $100 \%$ bias reported for Japanese E. hecabe (Narita et al., 2011), but incomplete transmission of sex-distorting symbionts is not unusual for lepidopterans. Second, there was great disparity in brood sex ratio within biased lineages. This was clearly evident in the 2008 P5 lineage, where 
daughters from an F1 brood of 5:19 (M:F) produced both unbiased (9:10) and biased (2:24) families, with F3 broods from the latter family ranging from female to male bias (5:32 and 6:24 vs 30:13; Figure 5). Hence, strong offspring bias in this lineage persisted alongside high inter-brood variation over two generations of inheritance. This could either reflect inconsistent transmission of a sex distorter or a variable efficacy of sex distortion depending upon host nuclear genome (sensu. Kageyama et al., 2009). The latter possibility includes a potential role of paternal genotype, which could be informed by pedigree-based approaches such as backcrossing.

\section{Antibiotic inversion of brood sex ratio}

Both experiments demonstrated antibiotic effects only in broods of sex-biased pedigree. At the group level, treated dams overproduced sons to a degree equivalent to the overproduction of daughters by untreated cousins. This implicates a causal role for intracellular bacteria, as opposed to protozoans, viruses or fungi (Kageyama et al., 2012). It also discounts causality based purely upon nuclear agents such as meiotic drivers (without excluding their interactive role; sensu. Kern et al., 2015). Here, we discuss treatment effects in relation to bacterial sex distortion, given that two candidate mechanisms for diplodiploid arthropods-male killing versus feminization-make fundamentally different predictions.

Under a simple male-killing scenario, antibiotic treatment is expected to restore the viability of sons and thereby 1:1 sex ratios in subsequent broods. This is supported by studies of Lepidoptera generally (for example, Hurst et al., 1999; Charlat et al., 2007a; Tabata et al., 2011) as well as one wCI-infected Eurema population (Narita et al., 2007b). Alternatively, treatment of hosts subject to genetic feminization should completely invert offspring sex ratio because such individuals possess karyotypes that are either genetically male (ZZ) or require Wolbachia to achieve female phenotypic development (Kern et al., 2015). All-male broods have been repeatedly induced in doubly infected Japanese lines (Hiroki, 2002; Narita et al., 2007c, 2011). Against this background, our findings are intriguing because rather than generating categorically 50 or $100 \%$ sons, antibiotic treatment instead shifted offspring phenotypes quantitatively in the direction of male bias. This was equivalent, albeit with high brood-level variation, across experiments and despite antibiotic treatments of varied known potency (Charlat et al., 2007a).

We consider two explanations for this finding. First, son overproduction in antibiotic lines may reveal an underlying signature of host counteradaptation. This could arise via adaptations in feminized matrilines to compensate for their overproduction of daughters. Second, as reported in Ostrinia (for example, Kageyama and Traut, 2004), selected matrilines of Australian E. hecabe may harbor a male-killing endosymbiont that is also essential for the development of viable female phenotypes. Antibiotic treatment of these lines would rescue sons yet kill daughters, thereby generating male bias. However, to explain our results, mortality would have to be lower than $100 \%$ both for sons in female-biased lines and then daughters under antibiotic treatment.

Ultimately, distinguishing between male killing and feminization will hinge upon whether phenotypes from biased lines actually possess W chromosomes. An absence of cytogenetically-visible heterochromatin is uncharacteristic for Eurema except in putatively feminized Japanese matrilines (Hiroki, 2002; Narita et al., 2011; Kern et al., 2015). Such phenotypes are thought to either possess a degraded version of the $\mathrm{W}$ chromosome (that is, a W'Z karyotype) or lack the chromosome altogether (that is, 0Z; Kern et al., 2015). Our findings raise the possibility for a broader loss/degradation of the $\mathrm{W}$ chromosome in Australian E. hecabe, which could result from the population-wide invasion of a feminizer (Cordaux et al., 2011). This is known for isopod populations, wherein sexual phenotype is entirely determined by the presence or dosage level of feminizing Wolbachia inherited by host individuals (Rigaud et al., 1997).

\section{Potential causality of Australian sex bias}

On the basis of the absence of $w$ Fem, we conclude that sex bias in Australian E. hecabe does not arise simply as a geographic extension of Japanese feminization. The Wolbachia strain that we did identify $(w \mathrm{CI})$ induces cytoplasmic incompatibility in Eurema and Colias (Narita et al., 2007c, 2009, 2011). This wsp sequence is also indistinguishable from a resident of $H$. bolina known formerly for varied degrees of male killing across the Western Pacific (Charlat et al., 2005). Notably, $w C I$ has also been linked to incomplete male killing in one Japanese Eurema population (resulting in 69-89\% daughters; Narita et al., 2007b). However, antibiotic treatment reverted sex ratio in such lines to $1: 1$, unlike the male-biased outcomes we observed, and W chromatin is clearly observable (Narita et al., 2007b).

The omnipresence of $w C I$ across years regardless of variation in sex ratio argues generally against its sex-biasing role. In particular, this strain would neither be expected among parents and progeny of unbiased lines, nor to have persisted post-antibiotic treatment (given the effect of such treatment on sex ratio). The latter result may reflect incomplete curing, which is known for Wolbachia-infected lepidopterans, given one generation of adult tetracycline exposure (Kageyama et al., 2003; Charlat et al., 2007a). Yet under this scenario, we should have observed a qualitatively different effect of tetracycline versus rifampicin, either in the incidence of $w \mathrm{CI}$ or in offspring sex ratio (for example, Charlat et al., 2007a). One possibility is that the wsp sequence has become incorporated into the E. hecabe genome (sensu. Hotopp et al., 2007). This would explain why molecular assays proved universally positive, while simultaneously obscuring the actual incidence of $w C I$ itself (if present) based on $w s p$ PCR/sequencing. Beyond $w \mathrm{CI}$, the candidates for sex bias range from novel strains of Wolbachia to additional sex distorters such as Spiroplasma, Cardinium, Rickettsia and Flavobacteria (Kageyama et al., 2012). More detailed molecular investigation will be necessary to assess the full complement and potential role of bacterial agents in Australian E. hecabe, and to further explore the possibility of lateral gene transfer.

\section{CONCLUSION}

Feminized Japanese Eurema emphasize a dichotomy of all-female versus 1:1 offspring, but our data indicate a skewed continuous distribution of sex ratio in Australia. We found an incidence of incompletely biased broods that persisted across generations and despite reversal by antibiotic treatment. Resolving the causal basis will require dedicated microbial study (as above) plus more detailed consideration of factors such as incomplete transmission, variable virulence and host nuclear effects (sensu. Kern et al., 2015). Heuristically, our data are novel for the study of arthropod sex distortion (as summarized comprehensively by Werren, 2008; Cordaux et al., 2011; Kageyama et al., 2012). The mechanisms and consequences of complete sex biases are well-documented, including their ability to drive females to near-fixation frequencies in lepidopteran populations (Charlat et al., 2005). More subtle degrees of sex distortion - as revealed in this study — are by definition less likely to draw attention, yet may exist more broadly than appreciated. 


\section{DATA ARCHIVING}

These data have instead been placed in the Dryad repository. The DOI for this submission is: doi:10.5061/dryad.44t2n.

\section{CONFLICT OF INTEREST}

The authors declare no conflict of interest.

\section{ACKNOWLEDGEMENTS}

We thank Francis Jiggins, Nina Wedell and three reviewers for constructive comments, Yi San Leong for PCR testing and Scott O’Neill for supporting the molecular work. DJK was supported by the Australian Research Council (DP0557190 and DP140104107) and The Australia-Pacific Science Foundation (APSF10-09). FET was funded by James Cook University.

Charlat S, Davies N, Roderick GK, Hurst GD (2007a). Disrupting the timing of Wolbachiainduced male-killing. Biol Lett 3: 154-156.

Charlat S, Hornett EA, Dyson EA, Ho PPY, Loc NT, Schilthuizen M et al. (2005). Prevalence and penetrance variation of male-killing Wolbachia across Indo-Pacific populations of the butterfly Hypolimnas bolina. Mol Ecol 14: 3525-3530.

Charlat S, Hornett EA, Fullard JH, Davies N, Roderick GK, Wedell N et al. (2007b). Extraordinary flux in sex ratio. Science 317: 214-214.

Charlat S, Reuter M, Dyson EA, Hornett EA, Duplouy A, Davies N et al. (2007c). Male-killing bacteria trigger a cycle of increasing male fatigue and female promiscuity. Curr Biol 17: 273-277.

Cordaux R, Bouchon D, Greve P (2011). The impact of endosymbionts on the evolution of host sex-determination mechanisms. Trends Genet 27: 332-341.

Duplouy A, Iturbe-Ormaetxe I, Beatson SA, Szubert JM, Brownlie JC, McMeniman CJ et al. (2013). Draft genome sequence of the male-killing Wolbachia strain wBol1 reveals recent horizontal gene transfers from diverse sources. BMC Genomics 14: 20.

Dyson EA, Hurst GD (2004). Persistence of an extreme sex-ratio bias in a natural population. Proc Natl Acad Sci USA 101: 6520-6523.

Fisher RA (1930). The Genetical Theory of Natural Selection. 2 edn Clarendon Press: Oxford, UK.

Giorgini M, Monti MM, Caprio E, Stouthamer R, Hunter MS (2009). Feminization and the collapse of haplodiploidy in an asexual parasitoid wasp harboring the bacterial symbiont Cardinium. Heredity 102: 365-371.

Hamilton WD (1967). Extraordinary sex ratios: a sex-ratio theory for sex linkage and inbreeding has new implications in cytogenetics and entomology. Science 156: 477-488.

Himler AG, Adachi-Hagimori T, Bergen JE, Kozuch A, Kelly SE, Tabashnik BE et al. (2011). Rapid spread of a bacterial symbiont in an invasive whitefly is driven by fitness benefits and female bias. Science 332: 254-256.

Hiroki M (2002). Feminisation of genetic males by a symbiotic bacterium in a butterfly, Eurema hecabe (Lepidoptera: Pieridae). Naturwissenschaften 89: 167-170.

Hiroki M, Tagami Y, Miura K, Kato Y (2004). Multiple infection with Wolbachia inducing different reproductive manipulations in the butterfly Eurema hecabe. Proc Biol Sci 271: 1751-1755.

Hornett EA, Charlat S, Duplouy AM, Davies N, Roderick GK, Wedell N et al. (2006). Evolution of male-killer suppression in a natural population. PLoS Biol 4: 1643-1648.

Hornett EA, Duplouy AM, Davies N, Roderick GK, Wedell N, Hurst GD et al. (2008). You can't keep a good parasite down: evolution of a male-killer suppressor uncovers cytoplasmic incompatibility. Evolution 62: 1258-1263.

Hotopp JCD, Clark ME, Oliveira D, Foster JM, Fischer P, Torres MC et al. (2007). Widespread lateral gene transfer from intracellular bacteria to multicellular eukaryotes. Science 317: 1753-1756.

Hurst GDD, Jiggins FM, von der Schulenburg JHG, Bertrand D, West SA, Goriacheva II et al. (1999). Male-killing Wolbachia in two species of insect. Proc $R$ Soc Lond (B) 266 735-740.

Hurst GDD, Majerus MEN (1993). Why do maternally inherited microorganisms kill males? Heredity 71: 81-95.

Hurst LD, Atlan A, Bengtsson BO (1996). Genetic conflicts. Q Rev Biol 71: 317-364.

Jones RE (1992). Phenotypic variation in Australian Eurema species. Aust J Zool 40: 371-383.

Jones RE, Rienks J, Wilson L, Lokkers C, Churchill T (1987). Temperature, development and survival in monophagous and polyphagous tropical pierid butterflies. Aust J Zoo/ 35: 235-246.
Jiggins FM, Hurst GDD, Majerus MEN (1999). How common are meiotically driving sex chromosomes in insects? Am Nat 154: 481-483.

Jiggins FM, Hurst GDD, Jiggins CD, v d Schulenburg JH, Majerus ME (2000). The butterfly Danaus chrysippus is infected by a male-killing Spiroplasma bacterium. Parasitology 120: 439-446.

Kageyama D, Ohno S, Hoshizaki S, Ishikawa Y (2003). Sexual mosaics induced by tetracycline treatment in the Wolbachia-infected adzuki bean borer, Ostrinia scapulalis. Genome 46: 983-989.

Kageyama D, Traut W (2004). Opposite sex-specific effects of Wolbachia and interference with the sex determination of its host Ostrinia scapulalis. Proc Biol Sci 271: 251-258.

Kageyama D, Anbutsu H, Shimada M, Fukatsu T (2009). Effects of host genotype against the expression of Spiroplasma-induced male killing in Drosophila melanogaster. Heredity 102: 475-482.

Kageyama D, Narita S, Watanabe M (2012). Insect sex determination manipulated by their endosymbionts: incidences, mechanisms and implications. Insects 3: 161-199.

Kemp DJ (2008). Female mating biases for bright ultraviolet iridescence in the butterfly Eurema hecabe (Pieridae). Behav Ecol 19: 1-8.

Kern P, Cook JM, Kageyama D, Riegler M (2015). Double trouble: the combined action of meiotic drive and Wolbachia feminization in Eurema butterflies. Biol Lett 11: 20150095.

Maynard Smith J (1982). Evolution and the Theory of Games. Cambridge University Press: Cambridge, UK.

Narita S, Kageyama D, Hiroki M, Sanpei T, Hashimoto S, Kamitoh T et al. (2011). Wolbachia-induced feminisation newly found in Eurema hecabe, a sibling species of Eurema mandarina (Lepidoptera: Pieridae). Ecol Entomol 36: 309-317.

Narita S, Kageyama D, Nomura M, Fukatsu T (2007a). Unexpected mechanism of symbiont-induced reversal of insect sex: feminizing Wolbachia continuously acts on the butterfly Eurema hecabe during larval development. Appl Environ Microbiol 73: 4332-4341.

Narita S, Nomura M, Kageyama D (2007b). A natural population of the butterfly Eurema hecabe with Wolbachia-induced female-biased sex ratio not by feminization. Genome 50: 365-372.

Narita S, Nomura M, Kageyama D (2007c). Naturally occurring single and double infection with Wolbachia strains in the butterfly Eurema hecabe: transmission efficiencies and population density dynamics of each Wolbachia strain. Fems Microbiol Ecol 61: 235-245.

Narita S, Shimajiri Y, Nomura M (2009). Strong cytoplasmic incompatibility and high vertical transmission rate can explain the high frequencies of Wolbachia infection in Japanese populations of Colias erate poliographus (Lepidoptera: Pieridae). Bull Entomol Res 99: 385-391.

Negri I, Pellecchia M, Mazzoglio PJ, Patetta A, Alma A (2006). Feminizing Wolbachia in Zyginidia pullula (Insecta, Hemiptera), a leafhopper with an XX/XO sexdetermination system. Proc Biol Sci 273: 2409-2416.

Riegler M, O'Neill SL (2007). Evolutionary dynamics of insect symbiont associations. Trends Ecol Evol 22: 625-627.

Rigaud T, Juchault P, Mocquard JP (1997). The evolution of sex determination in isopod crustaceans. Bioessays 19: 409-416.

Rugman-Jones PF, Hoddle MS, Stouthamer R (2010). Nuclear-mitochondrial barcoding exposes the global pest western flower thrips (Thysanoptera: Thripidae) as two sympatric cryptic species in its native California. J Econ Entomol 103: 877-886.

Sokal RR, Rohlf FJ (1995). Biometry: The Principles and Practice of Statistics in Biological Research. W.H. Freeman and Company: New York, NY, USA.

Sugimoto TN, Ishikawa Y (2012). A male-killing Wolbachia carries a feminizing factor and is associated with degradation of the sex-determining system of its host. Biol Lett 8: 412-415.

Tabata J, Hattori Y, Sakamoto H, Yukuhiro F, Fujii T, Kugimiya S et al. (2011). Male killing and incomplete inheritance of a novel Spiroplasma in the moth Ostrinia zaguliaevi. Microb Ecol 61: 254-263.

Traut W, Sahara K, Marec F (2007). Sex chromosomes and sex determination in Lepidoptera. Sexual Dev 1: 332-346.

Trivers RL, Willard DE (1973). Natural selection of parental ability to vary the sex ratio of offspring. Science 179: 90-92.

Weeks AR, Marec F, Breeuwer JAJ (2001). A mite species that consists entirely of haploid females. Science 292: 2479-2482.

Weeks AR, Reynolds KT, Hoffmann AA, Mann H (2002). Wolbachia dynamics and host effects: what has (and has not) been demonstrated? Trends Ecol Evol 17: 257-262.

Werren JH (2008). Wolbachia: master manipulators of invertebrate biology. Nat Rev Microbiol 6: 741-751.

Zchori-Fein E, Perlman SJ (2004). Distribution of the bacterial symbiont Cardinium in arthropods. Mol Ecol 13: 2009-2016.

Zhou W, Rousset F, O'Neill S (1998). Phylogeny and PCR based classification of Wolbachia strains using wsp gene sequences. Proc Biol Sci 265: 509-515.

Supplementary Information accompanies this paper on Heredity website (http://www.nature.com/hdy) 